\title{
The impact of small lot ordering on traffic congestion in a physical distribution system
}

\section{KAMRAN MOINZADEH , TED KLASTORIN \& EMRE BERK}

To cite this article: KAMRAN MOINZADEH , TED KLASTORIN \& EMRE BERK (1997) The impact of small lot ordering on traffic congestion in a physical distribution system, IIE TRANSACTIONS, 29:8, 671-679, DOI: 10.1080/07408179708966377

To link to this article: https://doi.org/10.1080/07408179708966377

$$
\text { 册 Published online: } 31 \text { May } 2007 .
$$

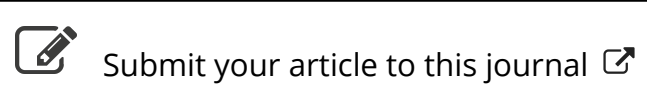

山 Article views: 64

Citing articles: 8 View citing articles 5 


\title{
The impact of small lot ordering on traffic congestion in a physical distribution system
}

\author{
KAMRAN MOINZADEH ${ }^{1}$, TED KLASTORIN' ${ }^{\prime}$ and EMRE BERK ${ }^{2}$ \\ ${ }^{1}$ Department of Management Science, School of Business, Box 353200, University of Washington, Seattle, WA 98195-3200, USA \\ 2 Faculty of Business Administration, Bilkent University, Ankara, Turkey
}

Received September 1995 and accepted April 1997

In recent years, some managers and researchers have advocated reducing lot sizes by decreasing setup costs, arguing that smaller lot sizes improve quality while reducing inventory levels and associated holding costs. However, smaller lot sizes result in an increased number of shipments which, in turn, exacerbates traffic congestion. This results in longer delivery times and, thereby, higher inventory levels. In this paper we study the relation between lot sizes and traffic congestion by constructing a model with numerous retailers who share a common congested delivery road. Using a numerical example, we illustrate the model's managerial implications with respect to several factors, including lot sizes, traffic congestion, and inventory levels. Our findings suggest that in a physical distribution system, if there are a relatively large number of retailers, no single retailer has an incentive to increase batch sizes because one retailer's effect on reducing traffic congestion will be negligible. If all retailers increase their lotsizes, however, traffic congestion will be reduced and all retailers will experience lower costs.

\section{Introduction}

In this paper we study the relation between lot sizes and traffic congestion in a physical distribution system. The idea of reducing lot sizes has been popularized in recent years as part of the 'just-in-time' (JIT) philosophy developed in the 1960 s by Ohno, and first implemented by Toyota [1]. JIT proponents advocate reducing setup costs to improve flexibility and the ability to adapt quickly to changing demand. It is further argued that smaller lot sizes (ideally a batch size of one) will result in reduced inventory levels requiring less warehouse space and lower costs (see Zipkin [2] for a more comprehensive discussion of JIT philosophy and practice).

Smaller lot sizes, however, lead to more frequent shipments, which increase traffic congestion and result in longer delivery lead times and higher inventory levels. In addition, increased traffic congestion causes environmental degradation and negatively impacts road conditions and social welfare (For example, vehicle carbon dioxide emissions increased from $15 \%$ in 1975 to about $20 \%$ in 1990 in Japan [3]. Also see NRC [4] for a discussion of related issues in the USA.). These costs are causing managers and government agencies alike to reconsider the theory and practice of small lot ordering.

Negative consequences of lot size reduction have been described in numerous recent articles [5-8]. Referring to the Tokyo metropolitan area, Shiomi et al. [3] state bluntly that "...the frequent movements of trucks under
JIT create chronic traffic jams in large cities and pollute the environment'. Abrahams [9] quotes the director of basic chemicals at one of Japan's largest plastics manufacturers, who stated that 'Acrylic sheet was infamous for small deliveries, even within the plastics industry. We were sometimes having to make deliveries three times a day. The costs were exorbitant. Not only did you have to cover the cost of freight, but you had to carry larger inventory too.'

In this research we study the impact of small lot ordering on traffic congestion in a supply chain. Specifcally, we consider a physical distribution system with a number of retail outlets that share a common road for deliveries (the Interstate-5 corridor in the Seattle metropolitan area is an example of such a situation). We assume that demand at the retail outlets is random, and that fixed order costs are zero. Furthermore we assume that the speed of the delivery vehicles on the common road is a non-increasing function of the number of the vehicles on the road (i.e., the traffic density). This latter assumption implies that order leadtimes are random and depend on the number of vehicles on the road at any point in time.

We initially develop expressions for the operating characteristics of the system. Using these expressions, we show that when JIT ordering creates traffic congestion, the retailers can reduce their operating costs through a coordinated effort by increasing lot sizes (the solution adopted by the Japanese plastics industry). When such a 
coordinated effort does not occur among retailers, there are implications for government agencies that can impose road fees (e.g., toll fees, usage fees, taxes). Such fees would reduce traffic congestion and its associated social, structural and environmental degradations while collecting money for road maintenance.

To the best of our knowledge this is the first work that studies the effect of small lot ordering on traffic congestion, order leadtimes and operating costs in a multi-location physical distribution system. Previous research in multi-echelon distribution system has studied systems where congestion is only a factor in the production process. In these situations, standard queueing models were used for modelling congestion [10-14]. Other related work includes studies between the batch size and production leadtimes in a single-facility production environment by Karmarkar [15] and Karmarkar et al. [16]. Assuming that production times are exponentially distributed, the authors modelled the production facility as an $M / M / 1$ queue and showed that production leadtimes increase as smaller production batch sizes are used.

The rest of this paper is organized as follows. In Section 2 we describe the physical distribution system and outline the relations which enable us to evaluate the operating measures of such systems. Specifically, we initially compute the steady-state probability distribution of the order leadtimes for our model. Using results from multiechelon inventory theory, we then find the probability distributions of the number of outstanding orders at each retailer and, subsequently, the average total expected cost rate. In the Section 3 we introduce the functional relation between the speed of the vehicles on the road and the traffic density. In Section 4 we present the results of a numerical experiment that illustrates the impact of traffic congestion on JIT ordering by using the model developed in previous sections. Finally, we summarize our findings and discuss possible extensions of the basic model.

\section{Specification of the physical distribution model}

Consider a physical distribution system consisting of $M$ retailers sharing a common delivery road for their goods. Without loss of generality, we assume that all retailers (with index set $I=\{1, \ldots, M\}$ ) stock one product (but not necessarily the same product) and order units from suppliers in integer multiples, $m_{i}$, of a minimum allowable order quantity, $b_{i}(i \in I)$. (The quantity $b_{i}$ is determined by packaging or freight considerations.) Inventories at all facilities are reviewed continuously and a $(Q, R)$ ordering policy is used at each location; that is, when inventory position $I_{i}$ (on-hand + on-order - backorders) reaches $R_{i}$, retailer $i$ places another order of size $Q_{i}$ [17], where $Q_{1}=m_{1} b_{1}$.

Demand for the product occurs at the retailers according to a Poisson process with a mean rate $\lambda_{i}$ for retailer $i$. If the retailer does not have sufficient units on hand to meet demand, a backorder occurs. For ease of exposition, we assume that retailers' orders are filled immediately by the suppliers (i.e., there are no order delays at the supplier). Therefore order leadtime is determined only by the transit time to ship $Q_{i}$ units from the supplier to retailer $i$. To simplify the discussion, we will use $Q$ to denote the vector of all order quantities (i.e., $\left.Q=\left(Q_{1}, Q_{2}, \ldots, Q_{M}\right)\right)$ and $\boldsymbol{R}$ to denote the vector of all reorder points (i.e., $R=\left(R_{1}, R_{2}, \ldots, R_{M}\right)$ ).

To study the impact of JIT ordering, we assume that fixed order costs are zero and that retailers order the minimum allowable batch size, $b_{i}$ (i.e., $m_{i}=1 \forall i \in I$ ). This assumption is consistent with the goal of JIT ordering [2] and the increased emphasis on EDI in supply chain management.

All orders are shipped on a common road to their respective retailers; the transit time on this road is denoted by the random variable $\tau$. The distance to be travelled by all vehicles on this common road is $Y$; vehicular speed is $v_{n}$ when there are $n$ vehicles on the road (i.e., $n$ is a measure of the traffic density). We assume that $v_{n}$ is a monotonically non-increasing function of $n$. The common road, however, may only constitute a portion of the total distance from the suppliers to each retailer. Thus we assume that there is an additional distance to each retailer; this latter segment is travelled on a non-congested road and takes a known time, $T_{i}(\forall i \in I)$. The total transit time (and, hence, the order leadtime) faced by retailer $i, \tau_{i}$, is then defined as $\tau_{i}=\tau+T_{i}$.

\subsection{Distribution of order leadtimes}

We note that traffic density is determined by the number of vehicles shipping goods to retailers as well as noncommercial traffic that may be using the common road. We will denote the average rate which the non-commercial traffic enters the common road by $\hat{\lambda}$; therefore the average rate of vehicle entry, $\lambda$, to the common road is equal to

$$
\lambda=\sum_{i \in I} \frac{\lambda_{i}}{Q_{i}}+\hat{\lambda},
$$

given that retailer $i$ orders in batches of $Q_{i}$. If the number of retailers in the distribution system is relatively large and/or the non-commercial traffic constitutes a significant portion of the total traffic to the road, the order arrival process to the road can be approximated by a Poisson process (see Feller [18] for a description, Albin [19] for extensive empirical tests that support the approximation, and Zipkin [10] for a general discussion and application of such an approximation). Therefore we approximate the entry of vehicles to the common road by a Poisson process with mean $\lambda$.

To derive the probability distribution of the number of vehicles on the road (and, hence, the probability distri- 
bution of order leadtimes), we let $x_{n}(t)=\left\{x_{1}(t), \ldots\right.$, $\left.x_{n}(t)\right\}$ denote the stochastic process that describes the status of the vehicles on the common road at time $t$ with $n$ vehicles on the road and $x_{i}(t)$ denotes the remaining distance to be travelled by the $i$ th vehicle on the road prior to $t$. Note that vehicles on the road are ordered on a FIFO basis; that is, $0 \leq x_{1} \leq x_{2} \leq \ldots \leq x_{n} \leq Y$. We derive expressions for the steady-state density of $x_{n}, p_{n}\left(x_{1}, x_{2}, \ldots, x_{n}\right)$, and the steady-state probability of having $n$ vehicles on the road, $P_{n}$, in the following proposition.

Proposition 1. If $\lim _{n \rightarrow \infty} n v_{n}>\lambda Y$, then the steady-state distributions for $x_{n}$ exists and

$$
p_{n}\left(x_{1}, \ldots, x_{n}\right)=\left(\lambda^{n} /\left[n ! v_{1} \ldots v_{n}\right]\right) K
$$

and

$$
P_{n}=\left[(\lambda Y)^{n} / n ! \prod_{i=1}^{n} v_{i}\right] K,
$$

where $K$ is a normalizing constant and is equal to

$$
K=\left\{1+\sum_{n=1}^{\infty}(\lambda Y)^{n} /\left[n ! \prod_{i=1}^{n} v_{i}\right]\right\}^{-1} .
$$

Proof: The details of the proof are described in Appendix A.

It is also shown in Appendix A that the Laplace-Stieltjes transform of the travel time $\tau$ of a vehicle on the common road, $L(\cdot)$, can be expressed in terms of the probabilitygenerating function of the number of vehicles on the common road, $M(\cdot)$, as follows:

$$
L[\lambda(1-z)]=M(z) \text {. }
$$

For certain forms of $v_{n}$ (for example, $v_{n}=\alpha / n$ for $n \geq 1$, where $\alpha$ is a constant), one can obtain closed form expressions for the steady-state probability distribution of the total travel time $\tau$ of a vehicle on the common road. Furthermore, from (4), the expected value and variance of $\tau$ are defined, respectively, as:

$$
E(\tau)=E(N) / \lambda
$$

and

$$
V(\tau)=[V(N)-E(N)] / \lambda^{2},
$$

where $N$ is the random variable denoting the number of cars on the common road.

Let $\delta_{i}$ denote demand during leadtime at retailer $i$. Since demand at each retailer is assumed to be Poisson, then $E\left[\delta_{i}\right]$ is equal to $\lambda_{i} E\left[\tau_{i}\right]$ on the basis of Little's law. But $\tau_{i}=\tau+T_{i}$; therefore

$$
E\left[\delta_{i}\right]=\left(\lambda_{i} / \lambda\right) E[N]+\lambda_{i} T_{i}
$$

by (5). Furthermore we have (see [20]):

$$
E\left[\delta_{i}\left(\delta_{i}-1\right)\right]=\lambda_{i} E\left(\tau_{i}^{2}\right),
$$

where $E\left(\tau_{i}^{2}\right)$ is the second moment of $\tau_{i}$. It follows that the variance of $\delta_{i}$ can be expressed as

$$
V\left(\delta_{i}\right)=\left(\frac{\lambda_{i}}{\lambda}\right)^{2} V(N)+\left(\frac{\lambda_{i}}{\lambda}\right)\left(1-\frac{\lambda_{i}}{\lambda}\right) E(N)+\lambda_{i} T_{i} .
$$

Let $B_{i}$ and $O H_{i}$ denote the number of units backordered and the number of units on-hand, respectively, at retailer $i$. To evaluate the steady-state operating measures at each retailer, we note that

$$
E\left(B_{i}\right)=E\left(\left[\delta_{i}-I_{i}\right]^{+}\right),
$$

where $(x)^{+}=\max (0, x)$.

Since $I_{i}$ is uniformly distributed in $\left[R_{i}+1, R_{i}+Q_{i}\right]$ [17],

$$
E\left(B_{i}\right)=\frac{1}{Q_{i}} \sum_{j=R_{i}+1}^{R_{i}+Q_{1}} \sum_{k=j}^{\infty}(k-j) g_{i}(k),
$$

where $q_{i}(\cdot)$ denotes the probability distribution of demand for retailer $i$.

Similarly, the expected on-hand inventory at retailer $i$ can be defined as follows:

$$
\begin{aligned}
E\left(O H_{i}\right) & =E\left(\left[I_{i}-\delta_{i}\right]^{+}\right)=E\left(I_{i}\right)-E\left(\delta_{i}\right)+E\left(B_{i}\right) \\
& =R_{i}+\frac{1}{2}\left(Q_{i}+1\right)-\lambda_{i}\left[E(N) / \lambda+T_{i}\right]+E\left(B_{i}\right) .
\end{aligned}
$$

In the absence of order costs, the expected total cost rate $T C_{i}\left(Q_{i}, R_{i}\right)$ at each retailer is given by the sum of average holding and backorder costs

$$
\begin{aligned}
T C_{i}\left(Q_{i}, R_{i}\right)= & h_{i}\left\{R_{i}+\frac{1}{2}\left(Q_{i}+1\right)-\lambda_{i}\left[E(N) / \lambda+T_{i}\right]\right\} \\
& +\left(h_{i}+\pi_{i}\right) E\left(B_{i}\right),
\end{aligned}
$$

where $h_{i}$ is the unit holding cost of an item at retailer $i$ per unit time, and $\pi_{i}$ is the unit backorder cost of an item at retailer $i$ per unit time. The expected total cost rate for the system, $T C(Q, R)$, is then the sum of the expected costs for all retailers; that is,

$$
T C(Q, R)=\sum_{i \in l} T C\left(Q_{i}, R_{i}\right)
$$

If the objective is to minimize $T C(Q, R)$, then, for fixed $Q$, the optimal reorder point at retailer $i, R_{i}^{*}$, is the largest value of $R_{i}$ that satisfies

$$
\sum_{j=R_{i}+1}^{R_{i}+Q_{i}} \sum_{k=j+1}^{\infty} q_{i}(k) \geq \frac{h_{i} Q_{i}}{h_{i}+\pi_{i}} .
$$

\section{Vehicular speed on the common (congested) road}

The relation between vehicular speed on a road and traffic density can be derived by considering the car-following behavior of single-driver-vehicle systems, or by directly studying the macroscopic behavior of platoons of vehicles 
on a given road section. As Gazis et al. [21] have demonstrated, the two approaches yield similar speed-density relationships. The relations obtained suggest that vehicle speed on a single road at steady state is a monotonic decreasing function of traffic density.

Greenshields [22] was among the first researchers to study the empirical relation between speed and traffic density and proposed an inverse relation approximated by a linear function. In order to better capture the true traffic behavior, especially for high traffic densities, several nonlinear (polynomial, logarithmic, and exponential) relation have since been developed. The particular choice of a relation depends on factors such as the geometry of the road segment and the nature of traffic flow. We refer the interested reader to Gazis [23], Gerlough and Huber [24] and Papageorgiou [25] for a summary of speeddensity hypotheses and supporting empirical data.

As Ross [26] observed, one shortcoming of previously suggested models is that the common speed for the isovelocic flow of traffic is either zero at some 'jam' density or approaches zero asymptotically as the traffic density increases. This assumption results in a non-ergodic queueing system in general and violates the stability condition stated in Proposition 1. Therefore we use a hybrid speed function similar to the one defined by Smulders [27] and supported by Ross [26]. In this case, vehicle speed declines linearly as a function of the number of cars at lower traffic density levels. If the traffic density surpasses a critical density level $n_{0}$, vehicle speed is reduced in hyperbolic fashion. The assumed relation between vehicle speed and traffic density can be described mathematically as

$$
v_{n}= \begin{cases}v_{\text {free }}-\alpha n, & 0<n \leq n_{0} \\ v_{0} n_{0} / n, & n>n_{0}\end{cases}
$$

where $v_{\text {free }}$ is the free, or equilibrium, vehicular speed as the traffic density, $n$, approaches zero, and $v_{0}$ is the speed at the critical density level $n_{0}$. To ensure continuity, we define

$$
\alpha=\frac{v_{\text {free }}-v_{0}}{n_{0}} .
$$

Proposition 1 specifies a stability condition that must hold for a steady-state distribution for $x_{n}$ to exist; that is,

$$
\lim _{n \rightarrow \infty} n v_{n}=v_{0} n_{0}>\lambda Y .
$$

On the basis of this condition, we let $\rho$ denote the ratio $\lambda Y / v_{0} n_{0}$, which provides an effective measure of utilization for the common road. We use this measure in the numerical example described in the following section.

Using (13) and the results obtained in the previous section, we can determine $P_{n}$, the probability distribution of the number of vehicles on the road. Let

$$
f(n)=\prod_{i=1}^{n}\left(v_{\mathrm{free}}-\alpha i\right)
$$

and

$$
g(n)=\frac{1}{n ! f(n)}-\frac{1}{n_{0} ! f\left(n_{0}\right)\left(n_{0} v_{0}\right)^{n-n_{0}}}
$$

Then

$$
P_{n}= \begin{cases}\left\{(\lambda Y)^{n} /[n ! f(n)]\right\} K, & n \leq n_{0} \\ \left\{(\lambda Y)^{n} /\left[n_{0} ! f\left(n_{0}\right)\left(v_{0} n_{0}\right)^{n-n_{0}}\right]\right\} K, & n>n_{0}\end{cases}
$$

where

$$
K=\left\{\sum_{n=0}^{n_{0}} g(n)(\lambda Y)^{n}+\frac{\left(v_{0} n_{0}\right)^{n_{0}+1}}{n_{0} ! f\left(n_{0}\right)\left(v_{0} n_{0}-\lambda Y\right)}\right\}^{-1} .
$$

The first two moments of the distribution of the number of vehicles on the road can then be expressed as

$$
E(N)=K\left\{\sum_{n=0}^{n_{0}} n g(n)(\lambda Y)^{n}+\frac{\lambda Y\left(v_{0} n_{0}\right)^{n_{0}+1}}{n_{0} ! f\left(n_{0}\right)\left(v_{0} n_{0}-\lambda Y\right)^{2}}\right\}
$$

and

$E\left(N^{2}\right)=K\left\{\sum_{n=0}^{n_{0}} n^{2} g(n)(\lambda Y)^{n}+\frac{\lambda Y\left(n_{0} v_{0}+\lambda Y\right)\left(n_{0} v_{0}\right)^{n_{0}+1}}{n_{0} ! f\left(n_{0}\right)\left(n_{0} v_{0}-\lambda Y\right)^{3}}\right\}$.

Using (16) and (17), we can calculate the mean and the variance of the number of orders outstanding at each retailer as defined by (7) and (8). Unfortunately, the specification of the probability distribution of the number of orders outstanding at each retailer $i, q_{i}(\cdot)$, is extremely complex when $n_{0}>1$. In this case, however, we can approximate the distribution of $q_{i}(\cdot)$ by using a two-parameter family. It can be easily shown that $E\left(\delta_{i}\right)<V\left(\delta_{i}\right)$. Therefore one alternative will be to approximate the distribution of $q_{i}(\cdot)$ with a negative binomial distribution with a mean and variance equal to $E\left(\delta_{i}\right)$ and $V\left(\delta_{i}\right)$ and evaluate the operating measures of the retailers using (10) and (11). The negative binomial distribution has been previously used to approximate the steady-state distribution of the leadtime demand at various sites in the multi-echelon inventory literature and has been shown to be effective (see Svoronos and Zipkin [11], Graves [28]).

Furthermore, the average speed on the road is given by

$$
\bar{v}=\frac{1}{1-p_{0}} \sum_{n=1}^{\infty} v_{n} p_{n}
$$

where $v_{n}$ is the vehicular speed when the traffic density is equal to $n$. Note that the term $1 /\left(1-p_{0}\right)$ appears because the average vehicular speed is calculated only for the instances when there are vehicles on the road.

The characteristics of the traffic model is illustrated in Fig. 1, which presents the relation between average speed, $\bar{v}$, and the road utilization ratio, $\rho$. This graph indicates that the average vehicle speed approaches zero asymptotically as the road utilization approaches unity (as ex- 


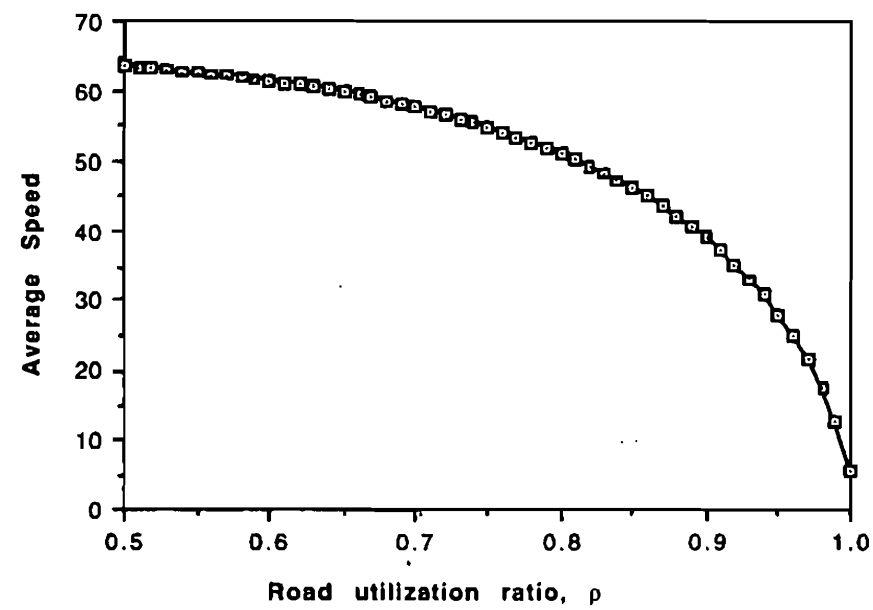

Fig. 1. Average speed, $\bar{v}$, plotted against the road utilization ratio, $\rho\left(v_{\text {free }}=70, v_{0}=60, n_{0}=5\right.$, and $\left.Y=5\right)$.

pected from the stability condition). For the value of $\rho=0.99$, which we use in the numerical example reported in the following section, Fig. 1 indicates that the average vehicular speed at that level of congestion is approximately $10 \mathrm{~km} / \mathrm{h}$.

\section{Numerical results}

In order to better illustrate the tradeoffs and managerial implications between lotsizing decisions and traffic congestion, we present the results of a numerical experiment where we analyzed 1000 scenarios. In each scenario we assumed that $M=30$ and that the retailers, having identical parameters, used equal lotsizes. For all retailers $i \in I$, we fixed $h_{i}=h=1$ and set the ratio $\pi_{i} /\left(\pi_{i}+h_{i}\right)=\pi /(\pi+h)$ to values $(0.90,0.95,0.98,0.99)$. Furthermore we varied the constant portion of the delivery leadtimes and set $T_{i}=T=(0.1,0.25,0.5,0.75,1.0)$. To allow for differences in product attributes, we varied the number of items in a minimum shipment, and set all $b_{i}=b=(1,5,10,25,50)$.

With respect to the common road, we set $v_{\text {free }}=70$, $v_{0}=60, n_{0}=5$, and $Y=5$. To investigate the impact of non-commercial traffic on road congestion, we varied the fraction of non-commercial traffic, $f_{\mathrm{NC}}$, on the common road from 0.50 to 0.95 in increments of 0.05 . Finally, the values of $\lambda_{i}$ (average demand at the $i$ th retailer) were set for all retailers at a value such that the road utilization ratio $\rho=\lambda Y / v_{0} n_{0}$ was 0.99 when JIT ordering was used (i.e., when $Q_{i}=b_{i}$ ). This implies that

$$
\widehat{\lambda}=0.99\left(n_{0} v_{0}\right) f_{\mathrm{NC}} / Y
$$

and

$$
\lambda_{i}=0.99 b n_{0} v_{0}\left[1-f_{\mathrm{NC}}\right] / M Y \quad \forall i \in I .
$$

This value of $\rho$ was chosen to highlight the traffic congestion effects as indicated in Fig. 1. It should be noted, however, that we varied $\rho$ in other numerical experiments; our results in all cases were similar to those reported here.

Using these parameters, we examined a number of performance measures under various conditions; results are presented in Figs 2 and 3. In Fig. 2 we compare the expected total cost $T C(Q, R)$ for JIT ordering (i.e., minimum batch sizes when $Q_{i}=b_{i}$ ) with optimal batch sizes for varying values of $f_{\mathrm{NC}}$. (In this case we set $T=0.75$, $b=10$, and $\pi /(\pi+h)=0.98$; however, similar results were found for other values of these parameters.) As the fraction of non-commercial traffic increases (i.e., as the contribution of the orders from the retailer group to the overall vehicle traffic decreases), the cost differential between JIT ordering (denoted in Fig. 2 by $T C_{\text {jit }}$ ) and optimal lotsizing (denoted in Fig. 2 by $T C_{\text {opt }}$ ) diminishes. This is as expected; as the fraction of non-commercial traffic increases, the impact of increasing lotsizes on leadtimes is reduced. It should also be noted that, although expected total cost decreases as a function of $f_{\mathrm{NC}}$, we are solving different inventory problems for each value of $f_{\mathrm{NC}}$. Therefore the significance of Fig. 2 lies in the relative difference between the expected costs of JIT and optimal ordering, and not the absolute values of the total costs.

Fig. 3 illustrates the impact of lotsizing as a function of the size of the constant portion of the delivery leadtime, $T$, when $f_{\mathrm{NC}}=0.70, b=10$, and $\pi /(\pi+h)=0.98$. (Again, similar results were found for other values of these parameters.) As $T$ increases, the impact of lotsizing gets smaller because traffic congestion and resulting travel time play a less significant role in the overall delivery delays. This has location policy implications for both retailers and suppliers; the supplier-retailer pairs that are closer to each other experience the biggest impact of the congestion on the road. However, as the distance between suppliers and retailers increases, the time on the con-

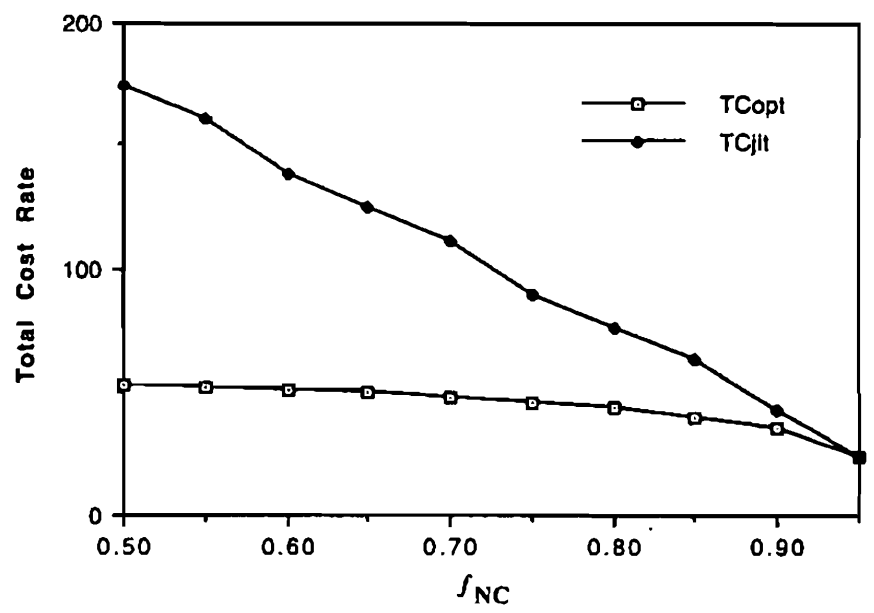

Fig. 2. Expected total cost for JIT ordering and optimal batch ordering $(T=0.75, b=10, \pi /(\pi, h)=0.98)$. 


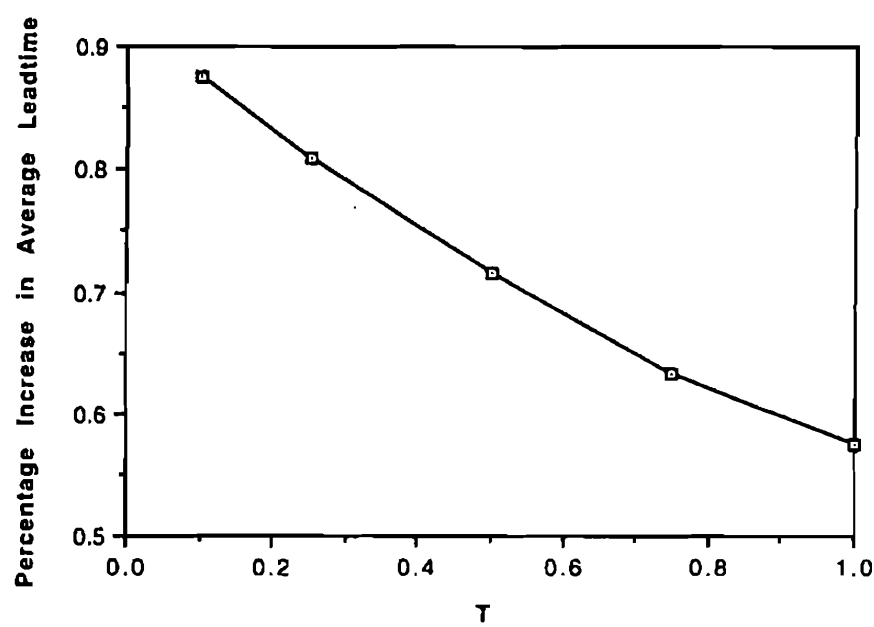

Fig. 3. Expected delivery leadtime, $E(\tau)$, plotted against the constant leadtime, $T\left(f_{\mathrm{NC}}=0.70, b=10, \pi /(\pi+h)=0.98\right)$.

gested road becomes a smaller fraction of the total leadtime, thereby having a smaller impact on batching decisions.

One of the cited benefits of JIT is that it reduces inventory and associated holding costs. To investigate the impact of congestion under JIT practice, we examined the on-hand inventory levels. In this case, $T=0.75, b=10$, and $\pi /(\pi+h)=0.98$. Fig. 4 illustrates the ratio of the on-hand inventory levels under JIT practice and optimal ordering as a function of the fraction of non-commercial traffic on the congested road, $f_{\mathrm{NC}}$. We note that the on-hand inventory levels are significantly lower when a retailer facing congestion deviates from JIT and orders in batch sizes larger than the minimum allowable shipment size.

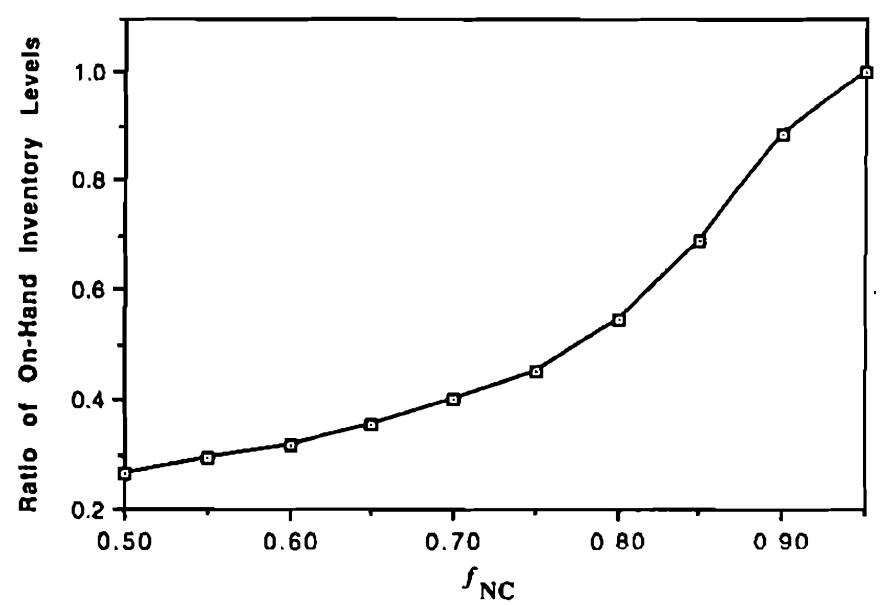

Fig. 4. Ratio of on-hand inventory levels under JIT practice and optimal ordering levels $(T=0.75, b=10, \pi /(\pi+h)$ $=0.98$ ).

\section{Conclusions and extensions}

In this paper we studied a physical distribution system where a number of retailers share a common congested road. Assuming that order costs are negligible, we first developed a model for the traffic flow on the congested road. Using results from multi-echelon inventory theory, we derived the probability distributions of the number of outstanding orders at each retailer and, subsequently, the average total expected cost rate.

Our results indicate that ordering minimum batch sizes, even when there is no fixed cost associated with placing an order, may not be optimal in terms of minimizing total expect cost when road congestion is present. Aside from the operating cost perspective of the retailers, traffic congestion has public policy implications as well. As the traffic intensity increases due to commercial use, the travel time of all vehicles on the road increases. Other negative externalities occur such as degradation in road conditions and environmental pollution.

It is interesting to note that, if there are a relatively large number of retailers, no single retailer has an incentive to increase batch sizes because one retailer's effect on reducing traffic congestion is negligible. If all retailers increase their lotsizes, however, traffic congestion is reduced and all retailers experience lower costs. Given the environmental benefits and other externalities, this may be justification for imposing tolls on certain congested roads. The model presented in this paper could be used as a starting point for determining the magnitude of such tolls.

\section{Acknowledgements}

This research was supported in part by research grants to K.M. and T.K. from the School of Business and the Program in Engineering and Manufacturing Management (PEMM) at the University of Washington, and the Burlington Northern/Burlington Resources Foundation. Additional support was provided by a grant from the Washington state Department of Transportation to the second author. The authors also acknowledge the anonymous reviewers for their helpful suggestions.

\section{References}

[1] Monden, Y. (1983) Toyota Production System, Industrial Engineering and Management Press, Norcross, GA.

[2] Zipkin, P. (1991) Does manufacturing need a JIT revolution? Harvard Business Review, January-February, 4-10.

[3] Shiomı, E., Nomura, H., Chow, G. and Niiro, K. (1993) Physical distribution and transportation in the Tokyo metropolitan area. The Logistucs and Transportation Review, 29 (4), 335-356.

[4] NRC (1995) Expanding Metropolitan Highways: Implications for Air Quality and Energy Use, Transportation Research Board, National Research Council, Washington, D.C. 
[5] Jackson, T. (1991) Flexible response to delivery dilemma comes just in time. The Independent, May 10.

[6] Takada, K. (1991) Just-in-time delivery sits at the crossroads. The Daily Yomiuri, May 12.

[7] Cullison, A.E. (1992) Congested roads in Japan thwart just-intime efficiency. Journal of Commerce, March 16.

[8] Bleakley, F. (1994) Just-in-time inventories fade in appeal as the recovery leads to rising demand. Wall Street Journal, October 25.

[9] Abrahams, P. (1994) Just in time now just too much - Japan's plastics suppliers are leading a revolt against the much praised management tool. Financial Times, March 30, p. 17.

[10] Zipkin, P. (1986) Design and control of stochastic, multi-item batch production systems. Operations Research, 34, 91-104.

[11] Svoronos, A. and Zipkin, P. (1988) Estimating the performance of multi-level inventory systems. Operations Research, 36 (1), 59-72.

[12] Deuermeyer, B. and Schwarz, L.B. (1981) A model for the analysis of system service level in warehouse/retailer distribution systems: the identical retailer case, in Multilevel Production/Inventory Control (TIMS Studies in Management Science vol. 16), Schwarz, L. (ed.), Elsevier, New York, pp. 163-193.

[13] Moinzadeh, K. and Lee, H.L. (1986) Batch size and stocking levels in multi-echelon repairable systems. Management Science, 32, 1567-1581.

[14] Lee, H.L. and Moinzadeh, K. (1987) Two-parameter approximations for multi-echelon repairable inventory models with batch ordering policy. IIE Transactions 19 (2), 140-149.

[15] Karmarkar, U. (1987) Lot sizes, lead times and in-process inventories. Management Science, 33, 409-418.

[16] Karmarkar, U., Kekre, S. and Kekre, S. (1985) Lotsizing in multiitem multi-machine job shops. IIE Transactions, 17, 290-298.

[17] Hadley, G.J. and Whitin, T.M. (1963) Analysis of Inventory Systems, Prentice Hall, Englewood Cliffs, N.J.

[18] Feller, W. (1971) An Introduction to Probability Theory and Its Applications, vol. 2, Wiley, New York.

[19] Albin, S. (1981) On Poisson approximations for superposition arrival processes in queues. Management Science, 28, 126-137.

[20] Marshall, K.T. and Wolf, R.W. (1971) Customer average and time average queue lengths and waiting times. Journal of Applied Probability, 8, 535-542.

[21] Gazis, D.C., Herman, R.S. and Potts, R.B. (1959) Car-following theory of steady-state flow. Operations Research, 7, 499-505.

[22] Greenshields, B.D. (1934) A study of traffic capacity. Proceedings of the Highway Research Board, 14, 448-474.

[23] Gazis, D.C. (ed.) (1974) Traffic Science, Wiley, New York.

[24] Gerlough, D.L. and Huber, M.J. (1975) Traffic Flow Theory. Transportation Research Board Special Report no. 165, Wash. ington, D.C.

[25] Papageorgiou, M. (ed.) (1991) Concise Encyclopedia of Traffic and Transportation Systems, Pergamon Press, Oxford.

[26] Ross, P. (1988) Traffic dynamics. Transportation Research Board, 22B, $421-435$

[27] Smulders, S. (1990) Control of freeway traffic flow by variable speed signs. Transportation Research, 24B, 111-132.

[28] Graves, S.G. (1985) A multiechelon repairable inventory item with one for one replenisment. Management Science, 31, 12471256.

[29] Gnedenko, B. and Kovalenko, I.N. (1968) Introduction to Queueing Theory, Israel Program for Scientific Translations, Jerusalem.

[30] Schmidt, C.P. and Nahmias, S. (1985) $(S-1, S)$ policies for perishable inventory. Management Science, 31, 719-728.

[31] Moinzadeh, K. (1989) Operating characteristics of the $(S-1, S)$ inventory system with partial backorders and constant resupply times. Management Science, 35, 472-477.

[32] Moinzadeh, K. and Schmidt, C.P. (1991) An $(S-1, S)$ inventory system with emergency orders. Operations Research, 39, 308-321.
[33] Courant, R. and John, F. (1989) Introduction to Calculus and Analysis, vol. 1, Springer-Verlag, New York.

[34] Stidham, S., Jr (1970) $L=\lambda W$ : a discounted analogue and a new proof. Operations Research, 20, 708-732.

[35] Cox, D.R. (1967) Renewal Theory, Methuen, London.

[36] Gross, D. and Harris, C.M. (1985) Fundamentals of Queueing Systems, Wiley, New York.

\section{Appendix A}

\section{Proof of Proposition 1}

Let $p_{n}\left(t, x_{1}, \ldots, x_{n}\right)$ denote the probability density of the road status's being $x_{n}$ at time $t$. We derive the system of partial differential equations and their boundary conditions that describe the status of the vehicles of the road. Our approach is similar to that employed by Gnedenko and Kovalenko [29], Schmidt and Nahmias [30], Moinzadeh [31] and Moinzadeh and Schmidt [32].

Case 1: $n=0$. Let $\Delta>0$ be a small number. In order to have no vehicles on the road at time $t+\Delta$, either there were no vehicles on the road at $t$ and no new vehicle entered the road during $(t, t+\Delta)$ or there was one vehicle on the road at time $t$ travelling at a speed $v_{1}$ with a remaining distance to be travelled less than $v_{1} \Delta$. This means that by time $t+\Delta$, the vehicle has reached its destination and therefore left the road. Thus we have:

$$
\begin{aligned}
p_{0}(t+\Delta, \cdot)= & (1-\lambda \Delta) p_{0}(t, \cdot) \\
& +(1-\lambda \Delta) \int_{0}^{\Delta v_{1}} p_{1}(t, \zeta) \mathrm{d} \zeta+o(\Delta) .
\end{aligned}
$$

Using the integral mean value theorem ([33], pp. 139143), we can write (A1) as:

$$
\left[p_{0}(t+\Delta, \cdot)-p_{0}(t, \cdot)\right] / \Delta=-\lambda p_{0}(t, \cdot)+v_{1} p_{1}(t, \varepsilon)+o(\Delta),
$$

where $0 \leq \varepsilon \leq \Delta$.

Letting $\Delta \rightarrow 0$ and assuming that a steady-state solution exists, we get:

$$
\lambda p_{0}(\cdot)=v_{1} p_{1}(0)
$$

Case 2: $n>0, x_{1}>0$ and $x_{n}<Y$. To be at $x_{n}$ at time $t+\Delta$, one of the following should happen:

(1) There were $n$ vehicles on the road at time $t$ with remaining distances to be travelled equal to $\left(x_{1}+\Delta v_{n}, \ldots, x_{n}+\Delta v_{n}\right)$ and there were no new entries to the road during $(t, t+\Delta)$.

(2) There were $(n+1)$ vehicles on the road at time $t$ with remaining distances to be travelled equal to $\left(\zeta, x_{1}+\Delta v_{n+1}, \ldots, x_{n}+\Delta v_{n+1}\right)$, where $0 \leq \zeta \leq \Delta v_{n+1}$, and there were no new entries to the road during $(t, t+\Delta)$. This means that the first vehicle on the road reaches its destination and leaves the road by $t+\Delta$ 
Thus, we have:

$$
\begin{aligned}
p_{n}(t & \left.+\Delta, x_{1}, \ldots, x_{n}\right) \\
= & (1-\lambda \Delta) p_{n}\left(t, x_{1}+\Delta v_{n}, \ldots, x_{n}+\Delta v_{n}\right)+(1-\lambda \Delta) \\
& \quad \times \int_{0}^{\Delta v_{n+1}} p_{n+1}\left(t, \zeta, x_{1}+\Delta v_{n+1}, \ldots, x_{n}+\Delta v_{n+1}\right) \mathrm{d} \zeta+o(\Delta) .
\end{aligned}
$$

Using the integral mean value theorem, adding and subtracting successive terms and letting $\Delta \rightarrow 0$ and $t \rightarrow \infty$, we get:

$$
\lambda p_{n}\left(x_{1}, \ldots, x_{n}\right)-\sum_{i=1}^{n} \frac{\partial p_{n}}{\partial x_{i}}=v_{n+1} p_{n+1}\left(0, x_{1}, \ldots, x_{n}\right) .
$$

Next we derive the boundary conditions for the above system of partial differential equations. Consider $\left[n, \zeta_{1}(t), \ldots, \zeta_{n}(t)\right]$ to be the position of a particle at time $t$ located in the region $0 \leq \zeta_{1}(t) \leq \ldots \leq \zeta_{n}(t) \leq Y$. The motion of the particle is discontinuous when a new delivery vehicle enters the road (a new order is placed). The boundary conditions are derived by considering such discontinuities. Define:

$$
\begin{aligned}
& Q_{n}\left(t, x_{1}, \ldots, x_{n-1}, Y\right) \\
& \quad=\int_{x_{1}-\delta}^{x_{1}} \ldots \int_{x_{n-1}-\delta}^{x_{n-1}} \int_{Y-\delta}^{Y} p_{n}\left(t, \zeta_{1}, \ldots, \zeta_{n}\right) \mathrm{d} \zeta_{n} \ldots \mathrm{d} \zeta_{1},
\end{aligned}
$$

where $\delta \geq 0$ is a small number. $Q_{n}\left(t, x_{1}, \ldots, x_{n-1}, Y\right)$ represents the probability mass in a neighborhood of the boundary $\left(x_{1}, \ldots, x_{n-1}, Y\right)$.

Applying the mean value theorem, for some $0 \leq \varepsilon_{i} \leq \delta(i=1, \ldots, n)$, we get:

$$
Q_{n}\left(t, x_{1}, \ldots, x_{n-1}, Y\right)=\delta^{n} p_{n}\left(t, x_{1}-\varepsilon_{1}, \ldots, Y-\varepsilon_{n}\right)+o\left(\delta^{n}\right) .
$$

Furthermore,

$$
\begin{aligned}
& Q_{n}\left(t+\delta / v_{n}, x_{1}, \ldots, x_{n-1}, Y\right) \\
& =\lambda \frac{\delta}{v_{n}} \int_{x_{1}-\delta+\delta\left(v_{n-1} / v_{n}\right)}^{x_{1}+\delta\left(v_{n-1} / v_{n}\right)} . \\
& \int_{x_{n-1}-\delta+\delta\left(v_{n-1} / v_{n}\right)}^{x_{n-1}+\delta\left(v_{n-1} / v_{n}\right)} p_{n-1}\left(t, \zeta_{1}, \ldots, \zeta_{n-1}\right) \mathrm{d} \zeta_{n-1} \ldots \mathrm{d} \zeta_{1} .
\end{aligned}
$$

Using the Taylor expansion together with the mean value theorem, $Q_{n}\left(t+\delta / v_{n}, x_{1}, \ldots, x_{n-1}, Y\right)$ can be also written as

$$
\begin{aligned}
& Q_{n}\left(t+\delta / v_{n}, x_{1}, \ldots, x_{n-1}, Y\right) \\
& \quad=\left(\lambda \delta^{n} / v_{n}\right) p_{n-1}\left(x_{1}-\varepsilon_{1}, \ldots, x_{n-1}-\varepsilon_{n-1}\right)+o\left(\delta^{n}\right) .
\end{aligned}
$$

Equating (A5) and (A6), dividing by $\delta^{n}$ and letting $t \rightarrow \infty$, we get:

$$
v_{n} p_{n}\left(x_{1}, \ldots, x_{n-1}, Y\right)=\lambda p_{n-1}\left(x_{1}, \ldots, x_{n-1}\right) .
$$

Assuming that a steady-state solution exists, a solution to the above differential equations and their boundary conditions is:

$$
p_{n}\left(x_{1}, \ldots, x_{n}\right)=\frac{\lambda^{n}}{n ! v_{1} \ldots v_{n}} K,
$$

where $K$ is the normalizing constant.

The steady-state probability of having $n$ vehicles on the road is now obtained as

$$
\begin{aligned}
P_{n} & =\int_{0}^{Y} \int_{x_{1}}^{Y} \ldots \int_{x_{n-1}}^{Y} p_{n}\left(x_{1}, \ldots, x_{n}\right) \mathrm{d} x_{n} \ldots \mathrm{d} x_{2} \mathrm{~d} x_{1} \\
& =\left[(\lambda Y)^{n} /\left(n ! \prod_{i=1}^{n} v_{i}\right)\right] K .
\end{aligned}
$$

Finally, the normalizing constant, $K$, is found by summing the probabilities to unity, which yields (3). For the steady state solution to exist, $K$ must be finite. This in turn means that

$$
\lim _{n \rightarrow \infty}\left(P_{n+1} / P_{n}\right)<1 .
$$

Substituting (A7) into (3) $\lim _{n \rightarrow \infty} n v_{n}>\lambda Y$ as the condition for the existence of the steady-state solution.

\section{Derivation of (4)}

To prove that (4) holds, we first focus on the times when a vehicle departs the road. Let:

$P_{n}^{(\mathrm{D})}=$ steady-state probability of having $n$ vehicles on the road as a vehicle departs the road

Then

$$
\begin{aligned}
P_{n}^{(\mathrm{D})}= & \frac{\begin{array}{c}
\operatorname{Pr}\{\text { a departure is about to occur and } \\
\text { there are } n+1 \text { vehicles on the road }\}
\end{array}}{\operatorname{Pr}\{\text { a departure is about to occur }\}} \\
= & \frac{v_{n+1} \int_{0}^{Y} \int_{x_{1}}^{Y} \ldots \int_{x_{n}}^{Y} p_{n+1}\left(0, x_{1}, \ldots, x_{n}\right) \mathrm{d} x_{n} \ldots \mathrm{d} x_{2} \mathrm{~d} x_{1}}{\sum_{i=0}^{\infty} v_{i+1} \int_{0}^{Y} \int_{x_{1}}^{Y} \ldots \int_{x_{i}}^{Y} p_{i+1}\left(0, x_{1}, \ldots, x_{i}\right) \mathrm{d} x_{i} \ldots \mathrm{d} x_{2} \mathrm{~d} x_{1}}
\end{aligned}
$$

Applying the results from Proposition 1, we get:

$$
P_{n}^{(\mathrm{D})}=\frac{\lambda v_{n+1}\left[(\lambda Y)^{n} /\left(n ! \prod_{j=1}^{n+1} v_{j}\right)\right] K}{\lambda \sum_{i=0}^{\infty} v_{i+1}\left[(\lambda Y)^{i} /\left(i ! \prod_{j=1}^{i+1} v_{j}\right)\right] K}=\frac{(\lambda Y)^{n}}{n ! \prod_{j=1}^{n} v_{j}} K=P_{n} .
$$


Therefore we conclude that the steady-state probability of having $n$ vehicles on the road at a departure point is equal to the steady-state probability of having $n$ vehicles on the road at an arbitrary point in time.

Because the vehicles leave the road on a FIFO basis, arrivals to the road are Poisson and, as shown in Proposition $1, P_{n}\left(x_{1}, \ldots, x_{n}\right)$ is independent of $\left(x_{1}, \ldots, x_{n}\right)$; then, from the law of total probability, we can express $P_{n}^{(D)}$ (and thus $P_{n}$ ) as:

$$
P_{n}=P_{n}^{(\mathrm{D})}=\int_{0}^{\infty} P_{n \mid t}^{(\mathrm{D})} \mathrm{d} F(t),
$$

where $P_{n \mid t}^{(D)}$ denotes the steady-state probability that a departing vehicle leaves $n$ vehicles on the road given that its travel time on the road was $t$ time units, and $F(\cdot)$ is the cumulative distribution function of time spent on the road, $\tau$, by a vehicle.

Furthermore, assuming that the process $x_{n}$, as defined before, is ergodic (that is, $\lim _{n \rightarrow \infty} n v_{n}>\lambda Y$ ), as shown in Stidham [34], Little's law must hold. Thus we conclude (Cox [35], pp. 45-46) that

$$
P_{n \mid t}^{\mathrm{D}}=\frac{(\lambda t)^{n}}{n !} \exp (-\lambda t)
$$

and

$$
P_{n}=\int_{0}^{\infty} \frac{(\lambda t)^{n}}{n !} \exp (-\lambda t) \mathrm{d} F(t)
$$

which, following Gross and Harris ([36], pp. 272-273), results in (4).

\section{Biographies}

Kamran Moinzadeh is the Burlington Northern/Burlington Resources Professor of Manufacturing Management, Professor of Management
Science, and the co-director of Program in Engineering and Manufacturing Management (PEMM) at the University of Washington. He received his B.A. in Computer Science from the University of California, San Diego, his M.S. in Operations Research, and his Ph.D. in Industrial Engineering from Stanford University. Dr Moinzadeh's publications have appeared in Management Science, Operations Research, Naval Research Logistics, European Journal of Operational Research, and IIE Transactions. His research interests include production/operations management, inventory and supply chain management, and quality management. Dr Moinzadeh is a member of INFORMS and IIE. He is currently the editor of the Department of Inventory in IIE Transactions and is serving as the Associate Editor for Operations Research. He has been the consultant to Microsoft Corp., Starbucks Coffee Inc., and Boeing Computer Services.

Ted Klastorin is the Burlington Northern/Burlington Resources Professor of Operations Management and Chair of the Department of Management Science (School of Business), and Adjunct Professor in the Department of Health Services (School of Public Health and Community Medicine) at the University of Washington, Seattle, Washington. Professor Klastorin also holds an appointment as senior research fellow at the $\mathrm{IC}^{2}$ Institute, The University of Texas, Austin, TX. He holds a B.S. degree from Carnegie-Mellon University (1969) and a Ph.D. from the University of Texas at Austin (1973). Professor Klastorin's research interests include logistics, quality control/assurance, project management, inventory, scheduling and queuing problems in manufacturing and service organizations. Current research projects include stochastic line balancing problems, resource allocation in project management, and the impact of the Internet on company performance. His recent articles have appeared in IIE Transactions, Journal of Applied Psychology, and Management Science. Professor Klastorin has consulted with numerous organizations, including Boeing, Microsoft, and Fluke Corp. He is a member of INFORMS and IIE, and serves as a member of the editorial boards of the Journal of Manufacturing and Service Operations and IIE Transactions.

Emre Berk is Assistant Professor at the School of Business, Bilkent University, Turkey. Dr Berk received his B.Sc. in Mechanical Engineering at Bogazici University, Turkey and his M.Sc. in Mechanical Engineering at Washington State University. He received his M.B.A. and Ph.D. in Operations Management at University of Washington. His research interests include supply chain management and stochastic models in health care applications. He is a member of INFORMS. 